\title{
GEORG SIMMEL: UNA TEORÍA CRÍTICA DE LA CULTURA
}

\author{
Eduardo Gutiérrez Gutiérrez \\ Universidad de Valladolid \\ eduardo.gutierrez.gutie@outlook.es
}

\section{Resumen}

El objeto principal del artículo es la exposición de la teoría crítica de la cultura de Georg Simmel, que podemos denominar «tragedia de la cultura moderna». A colación de esta exposición, de cuya actualidad no dudamos por cuanto se están poniendo sobre la mesa los problemas sociales y culturales que en buena medida todavía a día de hoy se padecen en las grandes urbes, presentamos a la idea de cultura de Simmel como condensación de la idea de cultura desarrollada por el idealismo alemán. Por otro lado, y como segundo objetivo, se tratará de demostrar la línea de continuidad que existe entre Simmel y la teoría crítica de la Escuela de Frankfurt en lo que respecta a la crítica a la cultura moderna.

Palabras clave: Simmel, cultura, teoría crítica, Escuela de Frankfurt, idealismo alemán.

\section{GEORG SIMMEL: \\ A CRITICAL THEORY OF CULTURE}

\section{Abstract}

The main object of the article is the exposition of the critical theory of culture of Georg Simmel, which we can call «tragedy of modern culture». In connection with this exhibition, whose topicality we do not doubt since the social and cultural problems that to a large extent are still suffered in large cities are being brought to the table, we present Simmel's idea of culture as a condensation of the idea of culture developed by German idealism. On the other hand, and as a second objective, we will try to demonstrate the line of continuity that exists between Simmel and the critical theory of the Frankfurt School with respect to the criticism of modern culture.

KeYwords: Simmel, culture, critical theory, Frankfurt School, German idealism. 


\section{LA IDEA DE CULTURA DEL IDEALISMO ALEMÁN A TRAVÉS DE SIMMEL}

Bien sea porque Simmel ha sido más recordado como sociólogo que como filósofo, bien sea porque su filosofía ha sido considerada como una suerte de pensamiento asistemático que proyecta el frenetismo de la vida moderna en las grandes ciudades, el caso es que su filosofía ha sido en muchas ocasiones reducida a filosofía de la cultura. Más aún, a una filosofía de la tragedia de la cultura moderna. Y si bien es cierto que la cultura es un concepto básico de su ontología, una entidad de primer orden junto a la vida, la reducción culturalista que se hace de su filosofía, a nuestro juicio, deja en mal lugar el resto de las teorías planteadas y de aportaciones realizadas para el futuro de la historia de la filosofía.

No obstante, no es el objetivo de este artículo la recuperación del pensamiento simmeliano ${ }^{1}$. Y aunque no vayamos a tratar este tema, este reduccionismo culturalista que se aplica sobre la filosofía simmeliana tiene mucho que ver con el objeto de que nos ocupa: en Simmel la idea de cultura adquiere una relevancia superlativa, hasta el punto de constituirse como el contrapunto dialéctico de la vida (das Leben), la idea nuclear de su metafísica de la vida. Y esta idea megárica de cultura, tal y como a continuación la expondremos, es la idea de cultura del idealismo alemán, o al menos una idea que recoge las características básicas desarrolladas por Kant, Hegel o Herder. Lo más interesante de esta continuación doctrinal, y donde a nuestro parecer radica la originalidad del artículo, es que no finaliza con Simmel, sino que, muy al contrario, Simmel reformula las implicaciones que esta idea megárica de cultura tiene para una teoría de la cultura incorporándola a los estudios que previamente realiza sobre la economía monetaria (Filosofía del dinero, 1900) y sobre la sociedad (Sociología, 1908), planteando una plataforma conceptual que será el caldo de cultivo de la teoría crítica de la Escuela de Frankfurt.

No hay espacio suficiente para exponer la metafísica de la vida de Simmel, sin la cual no es posible comprender su filosofía de la cultura. No obstante, y debido precisamente a esa importancia, diremos cuatro palabras al respecto.

La vida (das Leben), dice Simmel siguiendo muy de cerca el concepto de voluntad de Schopenhauer y Nietzsche (die Willen), es un torrente inmenso de energía que fluye a través de sus formas. Partimos de un estadio originario en el que la vida es pura voluntad sin límites, energía espiritual desatada. Como fluir incesante la vida no puede ser apresada; es lo que ella misma es en cada instante:

${ }^{1}$ A quien le interese el tema, he dedicado numerosos estudios y artículos a la cuestión simmeliana: «Un sociólogo moderno y un sociólogo de la modernidad» (2016); «El barbarismo de las masas visto desde la perspectiva de tres 'pensadores de lo social': Gustave Le Bon, Georg Simmel y José Ortega y Gasset», revista Ágora (2017) 36, pp. 101-132; «Georg Simmel: Una antropología filosófica», revista Contrastes (2018) 23, pp. 7-23; «Simmel y Ortega: En torno a la idea de filosofía como ejercicio de recepción y crítica», Revista de Estudios Orteguianos (2019) 39, pp. 262-264; y mi tesis doctoral: Simmel y Ortega: En torno a la idea de filosofía como ejercicio de recepción y critica (2019). 
una totalidad inaprehensible que se manifiesta a sí misma en cada uno de sus momentos, y que solo intuitivamente puede ser captada. Por eso la vida tiene deseo de darse una forma que le permita, de un lado, su manifestación empírica (bajo la forma de la realidad), y del otro, su perfeccionamiento (bajo la forma de la idea). Ese deseo de trascendencia de la vida es según Simmel (la influencia de la tesis volitiva de Schopenhauer es total) el principio primero de la realidad. Cuando la vida se da para sí una forma («autoconciencia»), crea un «más-vida» (mehr-Leben), que no es otra cosa que las formas objetivas de la ciencia, la religión, el arte, la política, la moral, la sociedad, etc., desde las cuales los seres humanos construyen y conocen el mundo. Pero sucede que las formas objetivas, en el momento en que se configuran a partir de la vida, adquieren una lógica autónoma en virtud de la cual se independizan de ésta y, en ocasiones, la someten; el "más-vida» se transforma en "más-que-vida» (mehr-als-Leben), poniendo fin al "ciclo natural» de trascendencia, que será también el ciclo de desarrollo normal de la cultura. En la historia, explica Simmel, son continuos los quebrantamientos de estos límites y el avance de la vida hacia nuevas formas de manifestación (el paso de la Edad Media a la Edad Moderna, o del feudalismo al monetarismo en su estado primitivo, son ejemplos históricos de este proceso).

De acuerdo con lo expuesto, podemos pensar en la metafísica de la vida de Simmel como una ontología dialéctica de la vida y la forma, dado que aunque la vida sea el principio primero de lo real, como apuntábamos arriba, no es sino en el enfrentamiento con la forma como ejerce su función fundacional de lo real. En este sentido, la ontología simmeliana está más próxima a Aristóteles que a Platón. Y en el contexto del idealismo del que nos ocupamos, media entre el vitalismo nietzscheano y el formalismo kantiano, como esa misma dialéctica ontológica pone de manifiesto. En este sentido, y de acuerdo con lo indicado acerca de la importancia sistemática de la filosofía de la cultura de Simmel, si no podemos hablar de una metafísica de la vida tampoco podremos hacerlo en los mismos términos de desconexión ontológica de una filosofía de la cultura: porque vida y forma o vida y cultural no se entienden desconectadas la una de la otra (desconexión que haría imposible la historia), no se puede interpretar la filosofía de la cultura sin la ontología dialéctica de la vida ni viceversa.

Si la vida encuentra en la forma objetiva su necesario contrapunto dialéctico, la cultura se interpretará como una de esas formas objetivas. Más aún, y aquí se presenta una de las problemáticas más interesantes de la teoría simmeliana de la cultura: ¿la cultura es una forma objetiva más, junto a la ciencia, la religión o la moral, o es la suma de todas ellas? Más aún: ¿es una forma objetiva en cuanto «más-vida» o en cuanto «más-que-vida»? Una respuesta rápida, lo cual no significa que precipitada, dado que iremos desarrollando los argumentos que la justifican, es que la idea de cultura de Simmel es una idea megárica, es decir, que la cultura en Simmel es el conjunto de todas las formas objetivas que la vida se da para sí en su proceso de trascendencia. Y esto, tanto cuando estas formas son todavía «más-vida» como cuando son «más-que-vida».

La idea de cultura de Simmel entronca directamente con la tradición del idealismo alemán por dos razones fundamentales. En primer lugar, porque Simmel 
entenderá la culura como «reino-3»², es decir, como conjunto de artefactos, saberes, técnicas, instituciones, etc., que los individuos crean por sí mismos y para los otros, y que luego vuelven a su vida para conferirle cierta objetividad. $O$ diciéndolo desde el kantismo, la cultura es moral y conocimiento. La cultura como orden superior de los productos de la actividad humana; es decir, como Espíritu objetivo. La cultura es la Objetividad, frente a la Subjetividad. En este enfrentamiento, la Objetividad se acabará manifestando contra la Subjetividad como Objetividad despiadada, tesis que la Escuela de Frankfurt articulará como "cultura afirmativa», «sociedad opulenta», «mundo administrado» o "cultura de masas». En segundo lugar, la Objetividad cultural se opone o marca límite con respecto a la Objetividad natural. Es decir, que si la cultura era antes la Objetividad frente a la Subjetividad, ahora lo es frente a la Naturaleza. Y sumadas ambas: la cultura es la objetivación concreta de la vida y de la conciencia, esto es, de la vida como fuente de realidad y del individuo en cuanto vida individualizada.

Partiendo de Hegel, o según la filosofía del espíritu de Hegel, Simmel entenderá la historia como la historia del espíritu, que se divide en tres grandes órdenes: el orden animal, el orden espiritual y el orden cultural, en el que como ahora veremos se produce una contradicción interna. La posibilidad de este proceso es debida a que el ser humano no es solamente animal, sino también espíritu. Y es como espíritu que se enfrenta a la naturaleza, negándola. El paso al orden cultural se produce cuando el progreso vital de los individuos alcanza una potencia capaz de configurar estructuras objetivas que son expresión exacta de las formas de manifestación y expresión de las energías espirituales. Es decir, cuando la acción del individuo crea una objetividad cuya consistencia sobrepasa el nivel meramente individual, y se consolida como una forma objetiva coherente en sí misma. Éste es el proceso de la vida trascendiéndose a sí misma. El fuego, la agricultura, el arte, la artesanía, la caza, las jerarquías sociales, la magia o la religión primitiva son las primeras formas de cultura, desde donde se puede explicar el paso al tercer orden y al inicio del viaje del espíritu.

Cultura es entonces el camino desde la unidad cerrada hasta la unidad desarrollada del espíritu por la mediación de la multiplicidad cerrada de artefactos culturales. Es una actividad teológica e histórica de "formación», en el sentido en el que Herder, en Ideas para una filosofía de la historia de la humanidad, habló de la cultura como Bildung. Pero cultura también es el ejercicio de perfeccionamiento de la cosa natural, como cultivo, según la terminología originaria (cultivare), o también según la aceptación de cultura como Kultur propuesta por Herder.

Volviendo a lo que decíamos sobre la vida y la cultura, si la cultura es el conjunto de formas objetivas, su relación con la vida va a ser dialéctica, tan dialéc-

${ }^{2}$ En ontología, Simmel distingue tres reinos que componen la realidad: el reino-1, de las entidades físico-químicas; el reino-2, de las entidades psicológicas; y el reino-3, de las entidades suprapsicológicas y a pesar de ello objetivas, como las leyes físicas, las normas, las distancias o los objetos geométricos. Como el lector ha podido intuir, esta división tripartida es muy similar a la que realiza Karl Popper, distinguiendo tres mundos, o la que realiza Gustavo Bueno en la ontología especial de su materialismo filosófico, esta vez con tres géneros de materialidad. 
tica como la relación entre la vida y la forma. Aquí es donde descansa la tesis de la «tragedia de la cultura», quizás la idea filosófica de Simmel que mayor reconocimiento y recorrido ha tenido durante el siglo xx. La puesta de manifiesto de esta relación intrínseca entre la cultura y la vida nos obliga a redefinir o a matizar el punto de conexión con el idealismo alemán en la medida en que la Objetividad, aunque enfrentada a la Subjetividad, es también ella misma subjetiva. La cultura es un reino tan vasto que se articula en el sistema simmeliano como un concepto dinámico y ambivalente (como casi todos sus conceptos fuertes): cultura es la objetividad, pero también la subjetividad que la crea. Precisamente por eso el proceso cultural, en el momento en el que llega a un grado superlativo de contradicción, es trágico: porque lo que niega y lo negado son una misma cosa. En caso contrario, explica Simmel, se trataría de un proceso «triste» (traurig).

En cuanto concepto ambivalente, «cultura» refiere a dos entidades complementarias. De un lado la "cultura objetiva», que son las formas culturales propiamente dichas o el resultado del proceso cultural, y la "cultura subjetiva», los propios sujetos creadores. El proceso cultural, que consiste en una dialéctica siempre abierta entre la cultura subjetiva y la cultura objetiva, seguirá un ritmo normal de desarrollo siempre y cuando la cultura subjetiva impere sobre la cultura objetiva. Es decir, siempre que la cultura cumpla con la función que tiene para la vida; siempre que el individuo pueda hacerla o hacer uso de ella (la cultura objetiva puede ser creación individual o herencia social) para su perfeccionamiento interno. $\mathrm{O}$ dicho de otro modo: siempre que el «más-vida» no concluya en «más-que-vida»: «[...] la cultura significa aquel tipo de perfección individual que sólo puede consumarse por medio de la incorporación o utilización de una figura suprapersonal, en algún sentido ubicada más allá del sujeto» ${ }^{3}$. O en otras palabras: «Puede considerarse la cultura como el perfeccionamiento de los individuos merced a la provisión de espiritualidad objetivada por la especie humana en el curso de la historia» ${ }^{4}$.

Como el lector puede comprobar, hasta ahora apenas hemos salido de la filosofía del espíritu de Hegel: como la "gran empresa del espíritu» hegeliano, que consiste en que el espíritu subjetivo sale de sí hacia lo objeto para volver a sí con conciencia de sí y para sí, el proceso cultural de Simmel consiste en que la Subjetividad crea la Objetividad para perfeccionarse. Y como esa Objetividad tiene un carácter de consistencia histórica, la empresa histórica del individuo consiste en salir hacia lo Objeto, el reino-3, para perfeccionarse y alcanzar la plenitud de sus potencias espirituales. Pero si tenemos en cuenta que la relación entre la vida y la forma siempre requiere la conversión del "más-vida» en "más-que-vida», porque la vida tiene que romper los límites que se ha dado para progresar y continuar su ritmo de desarrollo, el proceso cultural tiene un carácter trágico insalvable: la cultura objetiva terminará por imponerse sobre la cultura subjetiva, de manera que las formas

\footnotetext{
3 Georg Simmel, «El concepto y la tragedia de la cultura», Sobre la aventura. Ensayos filosóficos, Barcelona, Península, 1988, p. 213.

${ }^{4}$ Georg Simmel, La religión, Buenos Aires, Gedisa, 2012, p. 21.
} 
culturales no serán ya instrumentos para el perfeccionamiento humano, sino formas autónomas con una lógica de desarrollo interno que no responden a los intereses y necesidades de la vida, y que se imponen sobre ella. Ésta es la famosa etapa de la «tragedia de la cultura».

En este punto, si el proceso normal de cultura estaba, siguiendo una terminología kuhniana, en clara sintonía con Hegel, el periodo extraordinario o trágico nos aleja de él, aproximándonos a Marx: si para Hegel la salida del espíritu al mundo para la vuelta sobre sí mismo culmina de un modo positivo, con la realización de la Idea en lo Real y la realización del Espíritu absoluto, Simmel no solo advierte sobre la posibilidad de desvío, sino que además considera que ese desvío o esa estandarización perversa de la cultura objetiva sobre la cultura subjetiva es necesaria para una nueva y más potente revolución cultural o formal. Ramos Torre expresa con bastante nitidez esa relación a tres entre Hegel, Marx y Simmel:

En términos más analíticos, el argumento de fondo es que la cultura objetiva, en razón de la lógica autónoma de su desarrollo y a resultas de la falta de un mecanismo de armonización que asegure el entretejimiento de sus cambios con los de la cultura subjetiva y, al modo de la más optimista Ilustración, la emergencia del mejor de los mundos posibles, puede desarrollarse de tal forma que le resulte extraña, inasimilable y hostil a quienes deben nutrirse de ellas.

Dicho en dos palabras: en el proceso cultural se tiene que producir necesariamente la tragedia de la cultura. Esta tragedia de la cultura, para ir afinando conceptos, consiste en una "hipertrofia objetiva" y su consiguiente "atrofia subjetiva»: "Aquí acontece un tornarse-objetivo del sujeto y un tornarse-subjetivo de algo objetivo, acontecimiento que constituye lo específico del proceso cultural y en el que, por encima de sus contenidos particulares, se muestra su forma metafísica» ${ }^{6}$. Mientras la cultura objetiva crece más y más, dado que cada esfuerzo individual redunda en su contenido y en cada acercamiento hay algo de la subjetividad que queda preso de ella, la cultura subjetiva, que a duras penas puede alcanzar su ritmo de desarrollo, se atrofia; o mejor, las facultades intelectuales o espirituales en virtud de las cuales el individuo puede acceder al reino de la cultura se debilitan, resolviéndose éste incapaz de tal asimilación. La necesidad de la tragedia en el proceso cultural, momento en el cual se produce la ruptura con la tradición precedente y se abre el camino para la posterior recepción de la herencia simmeliana, tragedia que a continuación analizaremos, se puede explicar en parte por aquella resistencia que ciertas formas objetivas cristalizadas en "más-que-vida» o ciertos objetos hipertrofiados (objeto, aquí, en el sentido de objetividad) ejercen sobre la voluntad del individuo:

5 Ramón Ramos Torre, «Simmel y la tragedia de la cultura», Revista Española de Investigaciones Sociológicas, 89, 2000, p. 51.

6 Georg Simmel, «El concepto y la tragedia de la cultura», Sobre la aventura. Ensayos filosóficos, Barcelona, Península, 1988, p. 209. 
Hay obras humanas de una perfección inalcanzable a las cuales, precisamente a causa de esta redondez sin lagunas, no tenemos ningún acceso o que, por ello, no tienen ningún acceso a nosotros. Una obra semejante permanece, digámoslo así, en su lugar, desde el cual no cabe transportarla a nuestros dominios; es una perfección solitaria hacia la que quizá podemos dirigirnos, pero que no podemos llevar con nosotros para alzarnos en ella a la perfección de nosotros mismos?.

\section{LA CRÍTICA DE LA CULTURA: TRAGEDIA DE LA CULTURA MODERNA}

El proceso de desenvolvimiento histórico consiste según Simmel en una dialéctica entre la vida y la forma o la vida y la cultura. Y aunque parece seguir a pies juntillas el planteamiento hegeliano, introduce, postulándose más a favor de Marx, que ese desenvolvimiento no necesariamente tiene un carácter teleológico-progresivo, sino que ofrece una tendencia negativa de enajenación que, irresoluble o no, hay que tener en cuenta a la hora de estudiar el proceso cultural e histórico. La tragedia de la cultura es una posibilidad inmanente al propio desarrollo cultural, y además una posibilidad que se confirma sobre la realidad histórica una y otra vez. Por eso nos parece que la propuesta simmeliana es interesante para los estudios culturales contemporáneos, porque a través de ella podemos poner negro sobre blanco la idea de cultura de la tradición germana, desde el idealismo a la teoría crítica. Asimismo, la teoría de la cultura simmeliana también es una teoría de la historia, entendiendo por tal la historia del desenvolvimiento de las formas objetivas con respecto a la vida de la que surgen.

Imaginen que las formas objetivas son como bolas de nieve que se deslizan por la historia, que en nuestra metáfora equivaldría a la superficie ligeramente inclinada de una montańa nevada. Pensemos que esa superficie no es completamente lisa, sino que a cada paso la bola de nieve se topa con pequeños desniveles que aceleran su velocidad de caída. Pensemos ahora que esos pequeños desniveles son intentos recurrentes de comprensión de las bolas de nieve o de las formas objetivas por parte de los individuos, o bien nuevas aportaciones culturales que se suceden a lo largo del tiempo. Podemos pensar también en esos obstáculos como intentos por parte de las subjetividades de frenar el recorrido de esas grandes esferas de realidad que impiden una correcta manifestación de sus fuerzas vitales.

Como es evidente, a cada nuevo obstáculo la bola de nieve realizará un pequeño salto que, como decimos, acelera su velocidad de caída. Y en el momento en el que vuelve a tocar suelo firme, la bola se hace más grande. Se hace más grande porque incorpora aspectos de las subjetividades que hacen uso de ella (educación) o la aumentan (creación cultural). Esa bola de nieve o esas bolas de nieve (que caen en distintas pendientes, lo que da cuenta de su irreductibilidad) se hacen cada vez más

\footnotetext{
${ }^{7}$ Ibidem, p. 217.
} 
y más grandes. Más si cabe en un tiempo en el que se ha experimentado un progreso material y tecnológico brutal, como éste en el que vivimos. De esta forma, la bola de nieve se acabará consolidando como una Gran Máquina de lo Objeto, concepto que acuñamos para recoger en él los conceptos que los pensadores de la Escuela de Frankfurt emplean en su crítica cultural, como por ejemplo el de «industria cultural».

En lenguaje simmeliano, la bola de nieve descontrolada es el "más-vida» convertido en "más-que-vida», es decir, independiente de los requerimientos de la vida creadora. Y su descenso por la montaña nevada en contradicción con otras formas o con los propios individuos creadores es el decurso cultural, no siempre progresista (ni teleológico, lo cual significa también toda una revolución en los estudios culturales). Como dice nuevamente en «El concepto y la tragedia de la cultura»: «Esta oposición [entre la vida como permanente fluir y las formas como cristalizaciones de su devenir] aparece como la incesante e imperceptible [...] batalla del flujo de la vida contra la fosilización histórica y el entumecimiento formal de un contenido cultural dado, desencadenándose, así, el impulso interno del cambio cultural $»^{8}$.

Y como también hemos apuntado antes, la tragedia de la cultura tiene graves consecuencias tanto para la vida en cuanto complejo ontológico como para la humanidad o los colectivos humanos en cuanto agentes históricos. No solo porque las creaciones culturales ya no responden a intereses y necesidades puramente humanos, sino también y sobre todo porque como han alcanzando un nivel hipertrofiado de desarrollo, frente a la atrofia individual o subjetiva, no se puede predecir bajo qué nuevas formas culturales se perfeccionarán. No se puede, y aquí descansa la tragedia del proceso, porque esa perfección ya no depende de la vida, en último término, o de la cultura subjetiva:

Sin duda, en general es correcto que el lenguaje imagina y piensa por nosotros, esto es, que recoge los impulsos fragmentarios o ligados de nuestro propio ser y conduce a una perfección a la que éstos, incluso puramente para nosotros mismos, no habrían llegado en caso contrario. [...] Incluso en ocasiones sentimos el lenguaje como un poder natural extraño que falsea y mutila no sólo nuestras manifestaciones, sino también nuestras orientaciones más íntimas?

Si la cultura objetiva y la cultura subjetiva mantienen una relación dialéctica de contradicción y reciprocidad, la hipertrofia objetiva y la atrofia subjetiva también. Porque las creaciones culturales se hipertrofian, es decir, crecen más allá de sus posibilidades (que son las posibilidades de desarrollo de la cultura subjetiva en cierta época histórica), la cultura subjetiva se atrofia, porque no puede seguir ese ritmo de desarrollo, aun cuando lo necesite. Y por otro lado, si la cultura subjetiva se atrofia (en razón de malas cosechas, de malos gobiernos, de guerras, de cambios revolucionarios en la dirección de los Estados, etc.), la cultura objetiva se hipertrofiará nuevamente. Cuanta más perfección alcancen los productos de la cultura

\footnotetext{
8 Ibidem, p. 307.

9 Ibidem, p. 220.
} 
objetiva y menos capacidades de asimilación tengan los integrantes de la cultura subjetiva, más se acentuará la tragedia. Y por otro lado, cuanto más incapaces sean los segundos de incorporar los primeros a su vida y desarrollo, más libres quedarán los primeros de un desarrollo autónomo respecto de los segundos.

La dialéctica entre la cultura objetiva hipertrofiada y la cultura subjetiva atrofiada se puede explicar también como dialéctica entre medios y fines, o como proceso de conversión de los medios en fines. En este punto planteamos un salto hacia lo que veremos a continuación, que tiene que ver con la idea de que si bien la tragedia de la cultura es un proceso inherente a la historia, que ha sucedido y sucederá repetidamente, la tragedia de la cultura moderna es radicalmente distinta a todas otras revoluciones culturales habidas. Y ello debido a una serie de causas que a continuación expondremos. Explica Simmel en Las grandes ciudades y la vida intelectual (1903):

[El] desarrollo de la cultura moderna se caracteriza por la supremacía de aquello que podría llamarse el espíritu objetivo, sobre el espíritu subjetivo, es decir, tanto en el lenguaje como en el derecho, en la técnica de producción como en el arte, en la ciencia como en los objetos de la vida doméstica, está personificada la suma del espíritu, cuyo crecimiento cotidiano es seguido a distancia y de manera sólo muy imperfecta, por el desarrollo espiritual de los sujetos ${ }^{10}$.

\subsection{LA TRAGEDIA DE LA CULTURA MODERNA}

Decíamos que la preponderancia de la cultura objetiva sobre la cultura subjetiva es un hecho histórico inevitable. No obstante, anunciábamos, en la modernidad, en razón de sus características peculiares y únicas (características económicas, tecnológicas, políticas, sociales, psicológicas, etc.), esta preponderancia parece haberse consolidado bajo una nueva figura teórica que confirma el triunfo completo de la objetividad despiadada sobre la subjetividad creadora. Por eso Simmel cree necesario diferenciar la tragedia de la cultura como fenómeno histórico de primer orden, necesario para la marcha misma de la historia, de la tragedia de la cultura moderna, un hecho único en la historia de la humanidad: «En la sociedad moderna, la acumulación de conocimiento, de destrezas, de recursos técnicos, de la maquinaria de la civilización, ha llegado a un punto en el que una cantidad y variedad abrumadora de productos creados de manera automática $»^{11}$.

En la modernidad se produce un curioso hecho que no se ha dado nunca en la historia de la humanidad: las cosas que constituyen nuestro mundo objetivo (objetos, instrumentos, medios de comunicación, productos de la técnica, etc.) están

${ }^{10}$ Georg Simmel, Las grandes ciudades y la vida intelectual, Discusión II. Teorías sobre los sistemas económicos, Barcelona, Barral, 1978, p. 22.

${ }^{11}$ Gianfranco Poggi, Dinero y modernidad. La filosofía del dinero de Georg Simmel, Buenos Aires, Buena Vista, 2006, p. 162. 
enormemente cultivados, mientras que los individuos no lo están al mismo nivel que aquéllas. Esto mismo podría expresarse, según la terminología orteguiana, con las tesis de la «revolución de las cosas», de un lado, y la «revolución de las masas», del otro:

En cierto modo, la preponderancia de la cultura objetiva sobre la subjetiva en el siglo xix se puede explicar por el hecho de que el ideal de educación del siglo XviII se orientaba hacia una formación del ser humano, esto es, hacia un valor personal e interno que, en el siglo XIX, quedó reprimido por el concepto de 'formación' en el sentido de conocimientos objetivos y formas de administración ${ }^{12}$.

Si la idea de la «tragedia de la cultura» seguía la línea del materialismo histórico de Marx, la "tragedia de la cultura moderna», precisamente en razón de las particularidades de la modernidad (digamos, a partir de 1850), supone una extensión de la teoría del "fetichismo de la mercancía» del plano de la producción al plano del consumo. El fetichismo, dice Simmel en Filosofía del dinero, ya no es solo económico, la alienación ya no solo la padece el trabajador en cuanto trabajador, sino que se convierte en un fenómeno universal que le aliena también en un tiempo de ocio (más allá de las implicaciones anejas al concepto marxista de "plusvalía»). Como podemos intuir, es en esta extensión del materialismo histórico donde se opera el paso de Hegel a la Escuela de Frankfurt: «Si no podíamos realizarnos mediante el proceso de auto-actualización del trabajo, tal vez podíamos lograrlo yendo de compras» ${ }^{13}$. Del "cogito marxista», dice Stuart Jeffries, pasamos al "cogito consumista»; ya no es "trabajo, luego existo", sino "consumo, luego existo".

Igual que las mercancías se independizan de las fuerzas productivas y económicas y se imponen sobre ellas con una legalidad autónoma que condiciona los modos de producción, los productos culturales se imponen sobre las fuerzas culturales y determinan los modos de creación de la cultura. La cultura formal y vacía choca y se enfrenta con los individuos culturales que pretenden reorientar su desarrollo. Si antes los objetos culturales servían para la emancipación y liberación subjetiva, ahora son obstáculos con los que el individuo tropieza en su búsqueda desesperada de libertad individual. Dado que este orbe de objetividad y cosificación de lo humano actúa con autonomía, las posibilidades de creación y desarrollo personal de los individuos están cada vez más restringidas; es el propio proceso de producción el que determina los modos de producción ${ }^{14}$. El productor fabrica de acuerdo con las condiciones obje-

12 Georg Simmel, Filosofía del dinero, Granada, Comares, 2003, p. 582.

13 Stuart Jefrries, Gran Hotel Abismo: Biografía coral de la Escuela de Frankfurt, Madrid, Turner Noema, 2018, p.136.

${ }_{14}$ Simmel capta a la perfección este proceso en sus escritos sobre la moda. Las modas, que en origen son modas de clase que las clases altas de una sociedad crean para diferenciarse de las clases más bajas, y que cuando éstas se apoderan de ellas mueren como modas, es decir, como elementos de distinción social, son hoy en día administradas por la industria de la moda. En la industria de la moda ya no hay clientes, sino consumidores, mejor dicho, un gran consumidor abstracto, el mercado, dentro del cual la pluralidad de potenciales clientes son reducidos a estilos de vida estandarizados. En su origen, las modas eran creadas por las élites aristocráticas de una sociedad para diferen- 
tivas impuestas por el sistema abstracto de la producción, sin posibilidad alguna de intervención creativa o de participación personal. La personalidad del trabajador se atrofia, porque se ve obligado a realizar actividades mecánicas y monótonas de las que no se siente responsable efectivo. Además, no se reconoce en lo que produce porque no lo produce para su vida, sino para satisfacer las exigencias del mercado.

Vamos ahora a ver cuáles son las causas que convierten a la «tragedia de la cultura moderna» en un fenómeno histórico completamente distinto a cuanto el ser humano había vivido anteriormente. Después veremos la más alarmante consecuencia de este proceso: la perversión de la cultura en «industria cultural».

Hay tres grandes grupos de procesos sociales, culturales y de producción industrial que identificamos como las causas principales de la «tragedia de la cultura moderna», que hacen de ella un fenómeno singular y novedoso: la técnica y la tecnología, la economía monetaria y la imposición del dinero como Dios moderno, y la especialización y la división del trabajo.

La técnica y su relación con el ser humano ha sido un tema central en la filosofía de los últimos tiempos, hasta el punto de poder hablar, hoy, de una filosofía de la técnica o teoría de la técnica. Cabe destacar, por ejemplo, las reflexiones que Martin Heidegger y José Ortega y Gasset realizan en torno a los límites y problemas de la técnica, atribuyéndole un sentido metafísico que la vincula directa y originariamente con la condición humana. En textos como "La pregunta por la técnica» (Die Frage nach der Technik, 1953) o Filosofía, ciencia y técnica (colección de conferencias), del primero, y Meditación de la técnica (1939) o El mito del hombre allende la técnica (1951), del segundo, se habla de la técnica como la facultad puramente humana que permite, junto con la inteligencia, el desarrollo cultural de la humanidad. Pero tampoco podemos olvidar El hombre y la técnica. Una contribución a la filosofía de la vida, de Oswald Spengler, en 1931; y Las dos fuentes de la moral y la religión, de Henri Bergson, en 1932. Asimismo, conviene destacar la que consideramos la última gran aportación a la cuestión filosófica de la técnica, esta vez desde las bases del materialismo histórico, que es el libro de Luis Carlos Martín Jiménez Filosofía de la técnica y de la tecnología, publicado en el año 2018.

La tesis de partida que los cuatro autores comparten (excepto el último, cuya teoría se erige en oposición a, al menos, las de los dos primeros), sería la siguiente: la técnica está vinculada a la inteligencia humana en cuanto producto suyo; el ser

ciarse del vulgo, que poco a poco iba adoptándolas hasta que la moda perdía su sentido como elemento diferencial. Las modas, entonces, tenían un sentido sociológico. Ahora, en la era de la industria de la moda, con los grandes desfiles y las grandes marcas, las modas se fabrican mecánicamente, sin una conexión íntima con ciertas clases sociales o tipos humanos; el consumidor no es un individuo particular (salvando la alta costura a medida), sino un conjunto homogéneo de consumidores; y el artículo de consumo, por ende, no se crea pensando en tal o cual cliente, sino que es un producto impersonal que se diseña de acuerdo con estadísticas de ventas, tendencias, encuestas acerca de gustos, etc. 
humano como homo sapiens es también, o termina por convertirse, en homo faber que crea y fabrica ${ }^{15}$.

Salvando su valor para la reflexión metafísica, la técnica, tal y como queremos estudiarla aquí, nos plantea una batería de problemas vinculados con la relación que configura entre el ser humano y el mundo. Es en este punto donde divergen las opiniones: de un lado están quienes consideran que la técnica es un medio para la evolución del ser humano y su adaptación en el mundo, y del otro quienes defienden la perversión del empleo de la técnica, que puede conducir, y de hecho conduce, del dominio del ser humano sobre el mundo al dominio del ser humano sobre el propio ser humano. Heidegger, por proponer un argumento ad hominen, participaría en ambos grupos. Dicho de otro modo: la técnica, o nos ayuda a llevar a la naturaleza (y a nosotros mismos) a plenitud, o nos confirma como tiranos en un mundo que solamente sirve, al modo heideggeriano, como «estación de servicio» para nuestros intereses. Simmel, con Max Weber y adoptando una posición crítica con respecto a la técnica, muestra su preocupación por el advenimiento de una época tecnificada al extremo en la que la técnica es expresión de la imposición de lo objetivo e instrumental, abstracto e impersonal, sobre lo subjetivo y humano, enajenado.

Aparentemente, la técnica y la tecnología son medios para hacerle más fácil la vida al ser humano, y para confirmar su dominio sobre el mundo. Sin embargo, y dada la mayor velocidad de desarrollo del ámbito de lo objetivo con respecto al ámbito de lo subjetivo (de lo humano), terminan por imponerse sobre el sujeto creador (y «empleador») hasta tal punto que le marcan un nuevo ritmo de desarrollo asfixiante y alienante. Es decir, la técnica desarrolla una complejidad de conexiones teleológicas entre los intereses y los instrumentos para satisfacer estos intereses que confunde al individuo y hace que tome lo que es solo un medio como el fin último de su acción. Así se explica que la tecnología, que nace como un medio para satisfacer intereses humanos, se convierta en fin último que le impone nuevos intereses artificiales. Lo que a nosotros nos interesa es profundizar en esta idea de la «hipertrofia teleológica de la técnica» ${ }^{16}$.

Pensemos en una acción que no es del todo cotidiana (no todos ni todo el tiempo la practicamos), pero que sí es lo suficientemente primitiva como para dar cuenta del progreso acontecido: la siembra del huerto (obviamos el hecho de que la agricultura ya es una técnica). En los orígenes de la agricultura, el apero necesario

15 Recordemos, a este respecto, lo que decía Ortega sobre que el pensamiento es una acción. Asimismo, es abundante la literatura filosófica sobre el modo como hacemos cosas con las palabras (How to do Things with Words, John Langshaw Austin, 1962). Gustavo Bueno defiende, desde las bases del materialismo filosófico, que el pensamiento (filosófico y científico) consiste en un conjunto de operaciones sobre términos, de donde surgen los conceptos y las ideas.

16 Para explicar la hipertrofia teleológica es muy útil el concepto de «heterogonía de los fines» del filósofo alemán Wilhelm Maximilian Wundt. La tensión entre lo objetivo y lo subjetivo, o entre lo tecnológico y lo humano, alcanza un nivel tan extremo de confrontación que estalla en un sistema utilitarista en el que las formas objetivas que eran medios para el objetivo del perfeccionamiento humano se convierten ahora en fines; y lo que antes eran fines humanos se reducen a simples medios para los objetivos de las formas culturales. 
para la labranza es muy básico: una azada, una hoz, un trillo. Conforme la tecnología avanza, el conjunto de instrumentos para la actividad agrícola se amplía y diversifica. Por el momento, es cierto, esta extensión instrumental es favorable a los intereses y a las necesidades de los labriegos. Pero llega un momento en el que el salto tecnológico, de cuantitativo, pasa a ser cualitativo, y se convierte en un problema para el ser humano: averías, inversiones, adelantos tecnológicos, etc. Traducimos este ejemplo en la siguiente idea: el desarrollo de la técnica y de la tecnología amplía y complejiza la cadena de medios necesarios para la consecución de un determinado fin; esta hipertrofia genera unos sentimientos de angustia y vértigo hasta el punto de que, como decíamos arriba, el individuo termina por tomar lo que era medio como fin último. ¿Por qué? Porque el fin último, que estaba directamente vinculado a la vida humana y que daba sentido a cada uno de los medios, tan extensa es ahora la cadena de medios, se ha borrado de la conciencia del individuo: «El sistema de fines en la modernidad se ha complicado tanto que ha desaparecido el significado de la vida $\aleph^{17}$.

En esta situación de inversión instrumental no son los fines (humanos) los que hacen buenos a los medios (artificiales), sino los medios y sus respectivos usos los que hacen buenos a los fines. El ser humano está perdido entre una maraña de artículos que le salen al paso so pretexto de facilitarle la vida, cuando lo que sucede realmente es que son esos mismos productos los que se la dificultan (sin contar con la presión publicitaria y mediática, industrias éstas, la de la publicidad y la de los medios de comunicación, que juegan un papel decisivo en el desarrollo de las modas).

La hipertrofia teleológica de la técnica no hubiese sido posible sin un contexto social-económico-político en el que el dinero se ha impuesto como patrón de medida último. En un mundo desencantado como el que se abre en la modernidad, en el que (lo hemos visto) no hay asideros ni consistencia alguna, donde todo flota y lo sólido se disuelve en el aire, que diría Marx, el dinero es el nuevo Dios capaz de dar orden, predicción y solidez a lo líquido e incierto. Es el instrumento perfecto para gestionar una realidad caótica que adquiere orden en la medida en que asume las características fundamentales de la economía monetaria ${ }^{18}$.

17 Francisco Gil Villegas, «El fundamento filosófico de la teoría de la modernidad en Simmel», Estudios Sociólogos, 15-43, 1997, p. 41.

${ }^{18}$ En «El significado del dinero para el tempo de la vida», Simmel distingue la «economía natural» o primitiva, regulada por el trueque y caracterizada por la simpleza y la inmediatez de sus relaciones económicas, de la «economía monetaria», capitalizada por el dinero y cuyas transacciones, además de multiplicarse respecto a las de la economía natural, son más complejas, impersonales e indirectas. El paso de una a otra consiste en un proceso de autonomización de los productos objetivos respecto de la personalidad subjetiva ("tragedia de la cultura»); este proceso alcanza su punto álgido en la modernidad, momento en el que la unidad entre la personalidad y las objetividades a las que estaba vinculado el individuo medieval desaparece, de manera que la personalidad se forja desde sí misma, mostrando un amplio espacio de libertad, tanto interno como externo. Y los contenidos creados por la potencia vital del individuo adquieren, en su manifestación práctica, una objetividad que los independiza de los sujetos creadores. Y en Filosofía del dinero explica que en la economía natural, que llega hasta la época medieval, el valor de los objetos que entraban en la relación de intercambio era un valor objetivo que se le atribuye a cada uno de los objetos como valor en 
El dinero construye a su alrededor una objetividad abstracta que, a modo de entidad natural, se impone sobre los individuos. Esta objetividad abstracta es un mundo objetivo de cosas que desarrolla una lógica autónoma en virtud de la cual traduce todo aquello que es atravesado por sus procesos de intercambio (cosas, mercancías, individuos) en elementos cuantificados que solo de acuerdo con esa misma lógica adquieren sentido y significado. En esto consiste la idea de la «tiranía del Dios dinero» en la sociedad industrial frankfurtiana, directamente conectada con la conversión de los medios en fines.

El proceso de objetivación monetaria o de extensión del modelo de la racionalidad monetaria actúa nuevamente como una bola de nieve en el ámbito social y político: constituido como una inmensa objetividad impersonal de lógica autónoma, conforme arrastra nuevas realidades que tritura para después configurar según las leyes del cálculo y la cuantificación, surgen nuevos productos, nuevos modos de organización, relación social y producción, nuevos conocimientos e incluso nuevas formas de vida. Si nos centramos en el ámbito social, el dinero es la causa principal de la despersonalización y objetivación de las formas y relaciones sociales, $y$, por ende, de un comportamiento generalizado de indiferencia, reserva y abstracción de subjetividades. El dinero opera una desvalorización-revalorizativa, es decir, que agota el valor subjetivo de los objetos y de los sujetos para atribuirles un nuevo valor, esta vez objetivo e impersonal (esto es, no referido a una singularidad individual, sino a un proceso objetivo de compra-venta de mercancías). Y es en razón de este proceso que el dinero, antes medio para la consecución de ciertos objetos u objetivos, pasa a convertirse en el fin último de todo intercambio; no solo es la fuente de valor de los elementos que participan de un intercambio (a nivel social, relación), sino, además, el elemento mediador entre ambos, capaz de reducir las diferencias.

Otra de las razones por las cuales el dinero fomenta la objetivación de las relaciones sociales está vinculada a la idea de la independencia del «ser» con el «tener». El propietario de objetos cuyo valor no se mide en función del dinero (por ejemplo, un terrateniente o un coleccionista de sellos) tiene su personalidad (con sus intereses, deseos y necesidades) vinculada de forma directa a la propiedad y al hacer sobre esa propiedad; su actividad no es libre (dado que la posesión de esos objetos implica ciertas obligaciones que condicionan su ser: labrar la tierra o arrendarla, cuidar cada sello, calcular su valor de mercado, etc.). Pero si la propiedad se reduce a dinero, o lo que es lo mismo, cuando la propiedad ya no es un objeto particular, sino una entidad abstracta que permite la vinculación con una pluralidad de objetos efectivos y posibles, la independencia del «ser» con respecto al «tener» es absoluta. Con la posesión de dinero, el propietario puede obtener el producto del trabajo de la tierra o de una pequeña fábrica sin quedar vinculado en su hacer al tener la tierra o la fábrica; el ser del sujeto no se desarrolla en la dirección marcada por el hacer

sí y para sí. De este modo, el comerciante podía establecer un precio justo para cada producto según tarifas. Pero en la economía monetaria, el dinero es un valor abstracto que se aplica sobre cada producto otorgándole un valor para sí. 
sobre la tierra o sobre la fábrica, sino en todas las que quiera. Quien posee dinero posee potencialmente o se asegura la posesión futura de una pluralidad de objetos de valor equivalente al valor del dinero poseído.

En razón de esta curiosa paradoja planteada por Simmel, él mismo concluye que el dinero y la economía monetaria, por cuanto independiza el «ser» del «tener», es garantía de una mayor libertad y autonomía. Pero hemos de aclarar dos cosas: en primer lugar, esa progresiva libertad va pareja a una también progresiva dependencia (para con los poseedores de los objetos de deseo); y en segundo lugar, la libertad de la que hablamos es una libertad vinculada a la posesión, es decir, una libertad como ausencia de restricciones externas (libertad negativa). Es una forma de libertad vinculada a la capacidad de expansión del yo o de la personalidad respecto a objetos y sujetos ${ }^{19}$ : «La expansión del yo que implica la posesión del dinero ofrece un sentido muy peculiar; es la más completa que nos puede aportar una propiedad $»^{20}$.

Los procesos y mecanismos por los cuales actúa la economía monetaria sobre las relaciones sociales y políticas, que se podrían interpretar como una respuesta racional a un crecimiento desmedido de las sociedades durante la modernidad, confluyen en una fetichización del dinero que, finalmente, desemboca en su idolatría. El dinero, como un Dios, es punto de convergencia de opuestos y unidad de medida de todo lo que existe; es expresión de lo absoluto como conjunción de todo lo relativo, de lo necesario como punto de unión de lo contingente. Es elemento de alienación para el individuo moderno porque, una vez muerto Dios y habiendo sido la religión y la metafísica desprestigiadas y puestas bajo el foco crítico de la Razón, se convierte en elemento moderno de idolatría: racional, mensurable y cuantitativo. Para conectar con el siguiente punto, el dinero no solo es causa de la complejidad de la época y de la cultura, sino que además impele al desarrollo del proceso de división del trabajo: produce desplazamientos y transformaciones estructurales motivadas por la cosificación de las condiciones objetivas de la vida urbana (es decir, por la racionalización o intelectualización de la vida) que afectan a las formas de interacción entre los individuos. Los sujetos ya no se comportan como unidades centralizadas, sino como entidades fragmentadas e informes, y frente a esta situación de decadencia anímica los objetos culturales se abstraen y autonomizan.

En cuanto a la división del trabajo y antes de nada cabe señalar que, aunque por motivos de desarrollo sintético de ideas la causa de la división del trabajo es la última de las tres, su efecto sobre la sociedad es bastante más llamativo que sobre la cultura, dado que se aplica fundamentalmente en el ámbito de la producción y no tanto en el del consumo (en el que su presencia es menor). El proceso de división

19 El gran capitalista es más libre con respecto al obrero no cualificado porque, poseyendo un importante capital, suceden dos hechos: primero, que ningún objeto le ofrece resistencia para su posesión; y segundo, que puede imponer su voluntad sobre la del obrero, que necesita del salario que le ofrece éste para vivir.

${ }_{20}$ Georg Simmel, "Avatares del dinero en la relación ser y tener», Cultura líquida y dinero. Fragmentos simmelianos de la modernidad, Barcelona y México, Anthropos, 2010, p. 37. 
del trabajo ${ }^{21}$, estudiado por Marx desde la perspectiva de la producción, motiva la progresiva independencia del producto de trabajo con respecto al trabajador $\mathrm{y}$, en consecuencia, genera un estado de alienación en razón del cual el individuo que produce ya no se reconoce en el objeto producido. El producto, una vez finalizado, se incorpora a un mundo de objetividades regido por una lógica autónoma.

Pensemos en un artesano que fabrica sillas para sus clientes. Él mismo es quien se ocupa de todos los procesos de producción de las sillas, por lo que es plenamente consciente de cada una de ellas, e incluso de cada uno de sus clientes particulares. Se ve reflejado en lo que hace, y sus clientes, como diría Unamuno, guardan el recuerdo del artesano al usar su silla. Pero imaginemos que este artesano, tanta fama tienen sus sillas, tiene que ampliar su pequeño negocio para cumplir con la demanda creciente. Ya no es él solo quien fabrica las sillas, sino dos, tres, cuatro o cuatrocientos trabajadores a su cargo. Por mucho que el artesano-jefe enseñe con dedicación y mimo a los trabajadores, por mucho que revise cada una de las sillas producidas, ésas ya no serán sus sillas, no se siente identificado con y en ellas. Eso es lo que significa, desde la perspectiva marxista, la división del trabajo:

El hombre que produce, no un todo, sino un trozo sin propio sentido, sin propio valor, no puede verter su persona en la obra, no puede contemplarse en su trabajo. Entre la integridad del producto y la integridad del productor existe un nexo constante, como cumplidamente se puede ver en la obra artística, cuya unidad sustantiva exige un creador único y se rebela contra toda coordinación de labores especiales diferenciadas. En la producción especializada, el sujeto permanece como ajeno al trabajo ${ }^{22}$.

Primera consecuencia del proceso de división del trabajo: escisión de lo subjetivo y lo objetivo para la autonomización de lo segundo con respecto a lo primero. Pero todavía hay más. De nuevo, una paradoja: en la misma medida en que la división del trabajo ofrece mayor libertad al trabajador, que no tiene que ocuparse sino de una sola tarea cada vez más mecanizada, le ata a muchos más individuos (es decir, voluntades), de los que depende para que su pequeña tarea dentro del proceso de producción en cadena dé sus frutos. Una tercera consecuencia, que hemos podido deducir de la primera: en el desdoblamiento entre lo subjetivo y lo objetivo, lo objetivo adquiere "vida propia», o lo que es lo mismo, su sentido ya no está vinculado a la subjetividad creadora, sino a una oferta abstracta o a un cliente masivo abstracto. Por no hablar, por supuesto, de la alienación que el trabajo mecánico y repetitivo hasta el extremo produce en el trabajador. Digamos que acontece un proceso dialéctico de subjetivación de lo objetivo y de objetivación de lo subjetivo. Además, como el desarrollo de las fuerzas internas del trabajador en su puesto de trabajo, tan minúsculo dentro del gran proceso productivo en masa, es unilateral, mientras que

${ }^{21}$ Cuyo origen localizamos en la división tribal entre el trabajo físico y el trabajo intelectual, momento en que surgen las castas religiosas (chamanes, sacerdotes).

22 Georg Simmel, La religión, Buenos Aires, Gedisa, 2012, p. 25. 
el producto final se nutre de una pluralidad de subjetividades (tantas como trabajadores compongan la línea de producción), la bola de nieve se hace más y más grande mientras la subjetividad creadora se vuelve más y más pequeña.

La gama de productos culturales, artísticos, tecnológicos, científicos, etc., de la que el individuo moderno puede disfrutar es mucho más amplia que la correspondiente al individuo primitivo, precisamente por el efecto de bola de nieve que produce el desarrollo hipertrofiado de la Objetividad. Pero, de nuevo, esta aparentemente mayor libertad de elección se contrarresta con la mayor dependencia hacia estos propios productos, tan alejados de la subjetividad creadora se hallan (recordemos el carácter abstracto e impersonal de los procesos de producción en cadena, para un público masivo). Desde esta plataforma (la objetivación impersonal de la cultura según el proceso de la división del trabajo y el progreso de la técnica y la tecnología), concluimos la siguiente tesis, que es el punto de partida del siguiente punto: en la modernidad emerge una forma perversa de cultura, «cultura de masas», que ya no sirve para la liberación o elevación y el cultivo del individuo y de la sociedad, sino para su estupidización y alienación crecientes.

\section{LA TRAGEDIA DE LA CULTURA MODERNA Y LA ESCUELA DE FRANKFURT}

$\mathrm{La}$ «tragedia de la cultura moderna» de Simmel se puede interpretar desde los parámetros de la «industria cultural»o «mundo administrado», conceptos que resumen el análisis crítico de la cultura de la Escuela de Frankfurt. Esto, claro está, teniendo en cuenta la polisemia o policromía de enfoques, conceptos y conclusiones dentro de este nutrido grupo pensadores. A efecto de poner en claro nuestro actual objeto de estudio, nos centraremos en el análisis de la idea de cultura de Max Horkheimer, Theodor Adorno y Herbert Marcuse ${ }^{23}$.

En cualquier caso, y como prueba de la línea de continuidad que pretendemos demostrar aquí, no salimos del esquema de hipertrofia objetiva y atrofia subjetiva que expusimos en el punto anterior, y que podemos resumir con la siguiente cita de Simmel:

El esplendor y la grandeza de la cultura moderna muestra así una analogía con aquel reino resplandeciente de las ideas de Platón, en el cual, el espíritu objetivo de las cosas es verdadero en una realidad inmaculada, pero al que faltan los valores de las personalidades, que no pueden diluirse en las objetividades, una falta que

${ }^{23}$ No obstante, creemos que sería un tema de estudio aparte la relación entre Simmel, sobre todo en lo que respecta a la revisión del materialismo histórico que propone en Filosofía del dinero, que al parecer estaría falto de una preinfraestructura psicológica y cuasimetafísica, y el trabajo de fusión del psicoanálisis y el marxismo de Erich Fromm. 
no puede dejar de sentirse, a pesar de la conciencia del carácter fragmentario, irracional y efímero de la personalidad ${ }^{24}$.

O también, y adoptando ahora una perspectiva más marcadamente marxista, con el inicio de La obra de arte en la época de su reproducción mecánica, de Walter Benjamin, con la que confirmamos la tesis expuesta dos párrafos más arriba: «La transformación de la superestructura, más lenta que la de la infraestructura, ha necesitado más de medio siglo para que los cambios en las condiciones de producción acaben incidiendo en todos los ámbitos de la cultura» ${ }^{25}$.

Concretando, diremos que la industria cultural, en su relación con el dinero, se caracteriza, a nivel psicológico (atrofia de la cultura subjetiva) ${ }^{26}$, por la indiferencia hacia los objetos, todos reducidos a mercancía, e incluso hacia los sujetos mismos, es decir, de unos sujetos hacia otros: por la indiferencia con respecto a las cualidades específicas de cosas e individuos, que quedan reducidos a valor de cambio o funciones sociales. Los individuos buscan satisfacer sus necesidades con determinados objetos no por el valor que éstos tengan en sí mismos, sino porque resultan más baratos que otros, porque valen menos. En términos orteguianos, podemos decir que la «industria cultural» o la «cultura de masas» es resultado de la «rebelión de las cosas». Esta indiferencia no solo se aplica hacia o contra los otros y las cosas, sino también, y como señala Marcuse, hacia uno mismo: "[el individuo] encuentra su alma en su automóvil, en su aparato de alta fidelidad, su casa, su equipo de cocina ${ }^{27}$. La cosa le dice al individuo qué debe desear, y cómo: "La tecnología no solo modifica lo que los humanos pueden hacer; modifica a los humanos, los hace desear cosas que antes no sabían que existían ${ }^{28}$. La mímesis individuo-mercancía o individuo-sociedad es inmediata y automática, y genera la pérdida de espacio para el pensamiento crítico y negativo.

En la sociedad industrial avanzada se alcanza tal nivel de enajenación racionalizada que el individuo alienado es devorado por su existencia alienada, esto es, por la representación de sí mismo proyectada sobre el objeto que es fuente de su alienación. O en un ámbito social, y volviendo de nuevo a Simmel: la función social devora la personalidad interna. Las mercancías se crean con el objetivo de satisfacer los intereses y las necesidades de los poderes que dominan la máquina de represión, y para tal satisfacción es necesario que adoctrinen y manipulen promoviendo una falsa conciencia. Conforme los productos de la manipulación se van volviendo cada

24 Georg Simmel, Filosofía del dinero, Granada, Comares, 2003, p. 608.

25 Walter Benjamin, La obra de arte en la época de su reproducción mecánica, Madrid, Casimiro, 2010, p. 9.

26 Por lo que respecta a la cultura objetiva, el adjetivo «industrial» nos pone sobre la pista de la hipertrofia burocrática que critican.

${ }_{27}$ Herbert Marcuse, El hombre unidimensional, Barcelona, Seix Barral, 1969, p. 39.

28 Stuart Jefreries, Gran Hotel Abismo: Biografía coral de la Escuela de Frankfurt, Madrid, Turner Noema, 2018, p. 207. 
vez más útiles y asequibles para un mayor número de individuos, el adoctrinamiento se convierte en el modo de vida bueno; esto es, se estandariza como estilo de vida.

Esto por lo que respecta solamente a la industria del consumo de productos de moda, hogar o tecnología; pero si ponemos atención en la industria artística y cultural, el panorama es todavía más desolador. Walter Benjamin, por ejemplo, habla del proceso de «tecnificación» de la obra de arte, o lo que es lo mismo, de la reproducción técnica de la obra de arte, como causa de la pérdida del sentido trascendente y elevado, del espacio para la protesta, la imaginación, el deseo y la libertad de la obra de arte y de la creación artística. En la época de la «cultura industrial» el arte y la cultura ya no son remansos de paz y verdad, escenario para la liberación del individuo, sino nuevas formas de explotación consumista gracias a la reproducción técnica y la publicidad perversa que fomenta estilos de vida represivos.

Según Benjamin, una obra de arte tiene un aura de misticismo que surge cuando su creación y su contemplación se realizan de acuerdo con un cierto ritual ${ }^{29}$. Una vez que las obras se reproducen mecánicamente, es decir, para un público masivo y sin un proceso de creación personal y reflexivo, esa aura desaparece. Como ya no es necesario acudir a un museo o a un teatro para disfrutar de "Las tres sombras" (1886) de Auguste Rodin o del Carmen (1845) de Georges Bizet, la experiencia artística se vulgariza. Así, como señala Horkheimer, actividades como la lectura, la escritura, la pintura o el deporte se convierten en hobbies, vacías ya de todo sentido personal y trascendente. Los hobbies se comparten, cultivan y discuten como si fuesen puras actividades mecánicas, no ya predilecciones personalísimas y racionales para el cultivo de uno mismo.

Hay otro punto que conviene desarrollar, y que ya hemos mencionado más arriba: el arte sometido a la lógica de la reproducción técnica pierde su sentido negativo y de protesta, su carácter revolucionario. De este modo, el arte se pone al servicio de los intereses de dominación que pretenden el mantenimiento del statu quo: «Otrora una obra de arte aspiraba a decir al mundo cómo es el mundo: aspiraba a pronunciar un juicio definitivo. Hoy se ve enteramente neutralizada ${ }^{30}$. La pérdida del carácter revolucionario de la obra de arte no debe entenderse desde el punto de vista del artista creador, o no solamente, sino más notoriamente desde el punto de vista del espectador. Porque si, como decimos, la contemplación de una obra de arte ha perdido su mística, si podemos contemplar «El bufón don Sebastián de Morra» (1645) de Velázquez como quien mira un partido de fútbol o lee un cartel publicitario, difícilmente podremos reconocer y pensar mensaje de crítica y protesta: «La composición ha sido cosificada, convertida en una pieza de museo, y su represen-

${ }^{29}$ Por el lado de la creación, podemos hablar del particular ritual de cada artista a la hora de inspirarse para la composición de su obra, del ritual de los artesanos para la construcción de sus figuras, o incluso de la preparación de los poetas y rapsodas antes de recitar sus versos. Por el lado de la contemplación, el itinerario marcado por la dirección de los museos de arte son por sí mismos rituales que despiertan los sentimientos y emociones de los visitantes. Sin olvidar, por supuesto, los grandes templos que fueron los primeros cines americanos y europeos.

30 Max Horkheimer, Crítica de la razón instrumental, Buenos Aires, Sur, 1973, p. 50. 
tación se ha vuelto una ocupación de recreo, un acontecimiento, una oportunidad favorable para la presentación de estrellas, o para una reunión social a la que debe acudirse cuando se forma parte de determinado grupo " ${ }^{31}$.

Resumiendo, en la sociedad industrial moderna acontece un proceso de instrumentalización de la cultura, en este caso particular del arte, motivado en parte por la suma de los tres factores que hemos descrito y analizado en el apartado anterior: "[lo] que determina la colocabilidad de la mercancía comercial es el precio que se paga en el mercado y así se determina también la productividad de una forma específica de trabajo ${ }^{32}$. Cuando la industria (convenientemente acompañada de la publicidad y los mass media) penetra en el mundo de la cultura, la producción de obras culturales ya no está orientada a la expresión del sentido profundo de las cosas, como otrora pudiera estarlo, sino a la venta masiva y mecánica (impersonal) de productos para el consumo.

$\mathrm{La}$ «alta cultura», que es el modo de referir a la cultura en cuanto lugar para el cultivo de la intimidad y para el progreso del ser humano, degenera en «cultura de masas». Si antes la cultura, y dentro de ésta el arte, trascendía la realidad y en esa trascendencia ofrecía un escenario de liberación y desarrollo, hoy, sobrepasada como está por la realidad, pierde los valores superiores implícitos en sus contenidos. Adoptando la idea de Marcuse del «hombre unidimensional» podemos afirmar que la «alta cultura», desnuda de su halo de trascendencia, queda reducida a una dimensión, la de la realidad efectiva del statu quo: la tensión bidimensional se rompe en favor de la unidimensionalidad manipulada y represiva. La «alta cultura» convertida en "cultura de masas» pierde su valor de verdad porque esta «alta cultura», que es la cultura de la sociedad preindustrial y pretecnológica, funda su validez en la experiencia de un mundo idílico que ya no puede ser recuperado. Tal experiencia queda invalidada por el progreso tecnológico, capaz de realizar el ideal en la tierra. Por eso Marcuse concluye que la «alta cultura» es «una cultura retrasada y superada, y solo los sueños y las regresiones infantiles pueden recuperarla ${ }^{33}$. La alta cultura, desnuda de valores superiores y de elementos trascendentes, es incapaz de diseñar la imagen de una sociedad mejor; la dimensión trascendente de la realidad, de la que la cultura era portadora o al menos inspiradora, ha desaparecido. Solamente queda una única dimensión, la de la realidad efectiva que se sostiene en el rechazo de los valores culturales. Tal rechazo consiste no en la eliminación de estos valores, sino en su asimilación y reproducción en masa: «Conforme las grandes palabras de la libertad y realización son pronunciadas por los líderes de las campañas y los políticos, en las pantallas de la televisión, las radios y los escenarios, se convierten en sonidos sin sentido que lo adquieren sólo dentro del contexto de la propaganda y los negocios, la disciplina y el descanso ${ }^{34}$ ».

\footnotetext{
31 Op. cit.

32 Ibidem, p. 51.

33 Herbert Marcuse, El hombre unidimensional, Barcelona, Seix Barral, 1969, p. 89.

34 Ibidem, p. 87.
} 
Hasta ahora hemos hablado de "cultura de masas» y de «industria de la cultura", que no son sino dos precipitados conceptuales que derivan del análisis de una misma realidad desde diferentes perspectivas (la del consumo y la de la producción). Pero será interesante continuar con este análisis de la cultura moderna introduciendo un nuevo concepto, «cultura afirmativa», acuñado por Marcuse ${ }^{35}$.

La «cultura afirmativa» refiere a la cultura de la burguesía, que en su desarrollo ha motivado la escisión entre el mundo anímico y el mundo espiritual de la civilización. Esta cultura, según Marcuse, se caracteriza por la afirmación de la existencia de un mundo valioso al que todo el mundo puede aspirar, y superior al mundo real. Un mundo que nos convierte a todos en individuos solemnes. A priori esto es una buena noticia: la cultura se abre a todos los públicos, fomentando el desarrollo conjunto de la sociedad. No obstante, y como corroboró Nietzsche desde su ya lejano siglo XIx, la democratización de la cultura tiene un sentido profundamente negativo, por dos razones: primera, porque al abrir el consumo de la «alta cultura» al gran público, ésta se vulgariza y pierde su dimensión elevada y de protesta; y en segundo lugar, y ahora volviendo a Marcuse, porque la "cultura afirmativa» es solo una respuesta ideal y abstracta, ilusoria, dentro de una realidad en la que sigue existiendo una división tajante entre el mundo cultural, reservado para unos pocos con capacidades adquisitivas suficientes, y un mundo material, el del gran público, en el que el interés por la "alta cultura» es escaso. En una sola palabra, la "cultura afirmativa» es la respuesta idealista de la burguesía recién asentada en el mando del mundo moderno (La rebelión de las masas) ante las exigencias de igualdad real (material) entre todos los individuos: «A la penuria del individuo aislado responde con la humanidad universal; a la miseria corporal, con la belleza del alma; a la servidumbre extrema, con la libertad interna; al egoísmo brutal, con el reino de la virtud del deber ${ }^{36}$.

En relación con esta idea de la vulgarización del arte, o la supresión de la alta cultura, Daniel Bell habla en Las contradicciones culturales del capitalismo de un proceso de democratización del arte que tiene lugar a comienzos de la década de los sesenta.

Tan sincretista es la cultura moderna que ya no se puede hablar de alta y baja cultura, porque todo se hace uno. Todo, tanto las obras como los sentimientos o las experiencias que motivan, se iguala al valor más bajo. Para una sociedad de masas que potencia la participación pública y despoja a las élites cultas de su autoridad y exclusividad de juicio, un arte de masas democratizado y, según cómo se mire, vulgar e ingenuo:

La democratización del genio se hace posible por el hecho de que si bien uno puede discutir juicios, no se pueden discutir sentimientos. Las emociones generadas por una obra nos atraen o no, y los sentimientos de una persona no tienen más autoridad

\footnotetext{
35 Ibidem, p. 89.

${ }^{36}$ Herbert Marcuse, El carácter afirmativo de la cultura, Buenos Aires, El cuento de plata, 2010, p.17.
} 
que los de otra. Con la expansión de la educación superior y el crecimiento de una intelectualidad semicapacitada, además, se ha producido un cambio significativo en la escala de todo esto. Gran cantidad de personas que antes habrían permanecido ajenas a la cuestión ahora reclaman el derecho a participar en la empresa artística, no para cultivar su mente o su sensibilidad, sino para 'realizar' su personalidad ${ }^{37}$.

Estas tres ideas sobre la degeneración industrial de la cultura se pueden resumir en la idea de la "gran máquina de Lo Objeto», que ofrecemos como síntesis de la crítica simmeliano-frankfurtiana.

Primero de todo, podemos dividir el concepto en dos expresiones de profundo significado filosófico-cultural: "gran máquina» y «Lo Objeto». Ambas expresiones refieren a sujetos autónomos que conforman, en su síntesis conceptual, el nuevo sujeto cultural que emerge en el siglo xx en virtud de los procesos y los acontecimientos mencionados: "máquina» refiere a la estructura mecánica de producción y consumo masivos de los productos culturales, y "Lo Objeto» a la Objetividad que estos productos configuran bajo la figura de un «reino de objetividades» ${ }^{38}$. En consecuencia, por "gran máquina de Lo Objeto" entendemos la idea de "cultura objetiva" de Simmel en el momento justo en que se produce la "tragedia de la cultura», es decir, en el momento en el que el producto cultural y objetivo adquiere autonomía suficiente para superar y oprimir la subjetividad creadora, "cultura subjetiva». En la medida en que la "cultura de Lo Objeto" se extiende por todas las dimensiones de la vida humana, tanto en la producción y el consumo como en las relaciones sociales, económicas y políticas, las producciones intelectuales y los acontecimientos históricos, deviene en "gran máquina» que se constituye como horizonte último de los destinos sociales e individuales.

Muy similar es la idea de la cultura moderna como «sistema total» que Horkheimer y Adorno exponen en Dialéctica de la Ilustración. Hoy en día, dicen los autores, comprobamos que cada ámbito cultural está armonizado en sí mismo y con todos los demás: el sistema de la industria de la cultura. Significa esto que los sistemas de producción del capitalismo industrial han penetrado en los sistemas culturales; dicho de otro modo, que las formas de organización del trabajo ahora configuran también los tiempos de ocio, controlando al individuo como productor

37 Daniel Bell, Las contradicciones culturales del capitalismo, Madrid, Alianza Universidad, 1989, p. 133.

${ }^{38}$ Asimismo, para la clara comprensión de la «gran máquina de Lo Objeto» es muy interesante, a modo de introducción, vincularla con la idea de la «jaula de hierro» de Max Weber (huelga decir, a este respecto, que Weber nunca llegó a escribir una expresión semejante a iron cage en La ética protestante y el espiritu del capitalismo, que en alemán sería eiserner Käfig. Este concepto es debido a la interpretación realizada por Talcott Parsons en su traducción de 1930. La expresión original de Weber, que después Parsons tradujo como «jaula de hierro", era "caparazón duro como el acero»). La célebre «jaula de hierro» de Weber significa el resultado del proceso de burocratización del mundo moderno, que nos introduce en una realidad social hiperregulada e hiperadministrada en la que las relaciones sociales se funcionalizan, y en la que el espacio de autonomía y libertad racional del individuo es cada vez más restringido. 
y como consumidor. La cultura se ha visto invadida por la racionalidad técnica, que es la racionalidad del dominio mismo. El triunfo de la «gran máquina de Lo Objeto» es el triunfo de la homogeneización cultural global. Técnica y arte, cultura y producción; todo se ha convertido en un único y colosal sistema:

La dependencia de la más poderosa compañía radiofónica de la industria eléctrica, o la del cine respecto de los bancos, define el entero sector, cuyas ramas particulares están a su vez económicamente coimplicadas entre sí. Todo está tan estrechamente próximo que la concentración del espíritu alcanza un volumen que le permite traspasar la línea divisoria de las diversas empresas y de los sectores técnicos ${ }^{39}$.

El ser humano ya no es lo que hace, sino lo que posee. El individuo moderno se define como función social y como consumidor. El consumidor, dice José Miguel Marinas, es el sujeto racional moderno que el nuevo orden objetivo instaurado en el triunfo de la cultura objetiva modela; un sujeto-objeto o sujeto-sujetado que se relaciona con objetos y funciones, no ya con otros sujetos; un sujeto cuya libertad se mide por el grado de satisfacción de sus deseos. Lo peor, que este sujeto es el centro organizador a partir del cual se articula el mundo de cosas que le rodea y que entiende como medios para la satisfacción de sus deseos: «El consumidor es la omnipresente figura que determina todo aquello que acontece. La sociedad moderna aparece como una organización democráticamente regida por el consumidor» ${ }^{40}$.

Para terminar, podemos reinterpretar las ideas simmelianas de hipertrofia y atrofia según la terminología frankfurtiana para apuntar que a medida que el aparato tecnológico que sostiene la máquina de represión y administración de la sociedad gana en racionalidad, productividad y desarrollo técnico, más posibilidades de liberación de la servidumbre tienen los individuos y más reales son los medios y modos para conseguirlo. Pero no hay libertad, dice Marcuse, sin conciencia de la libertad. Y no hay conciencia de la libertad si hay un predominio de necesidades falsas y represivas que el individuo asume como propias. Entonces, aunque las condiciones objetivas sean idóneas para la liberación del hombre, como también pone de manifiesto el pensamiento dialéctico de Simmel, la represión extendida y administrada burocráticamente por la gran máquina socava aquellas necesidades que pueden traer la libertad y la autonomía mientras sostiene y legitima en su racionalidad el poder represivo de una sociedad opulenta que fomenta la satisfacción de necesidades innecesarias: «Bajo el gobierno de una totalidad represiva, la libertad se puede convertir en un poderoso instrumento de dominación ${ }^{41}$.

Aunque la idea de la individualidad todavía se mantiene viva, dice Horkheimer, se le enseña al individuo que para arreglárselas en el mundo tiene

39 Max Horkheimer y Theodor Adorno, Dialéctica de la Ilustración, Madrid, Trotta, 1994 , p. 168.

40 José Miguel Marinas, "Simmel y la cultura del consumo», Revista Española de Investigaciones Filosóficas, 89, 2000, p. 136.

${ }^{41}$ Herbert Marcuse, El hombre unidimensional, Barcelona, Seix Barral, 1969, p. 37. 
primero de todo que rechazar la idea de una realización personal máxima. Si quiere éxito, tiene que ser en sociedad y mediante la imitación de tipos sociales considerados como buenos. De este modo, el individuo está obligado al conformismo más estricto, al sometimiento a las reglas del juego:

Así como el nińo repite las palabras de su madre y el adolescente el comportamiento brutal de los mayores bajo cuya férula padece, así el altoparlante gigantesco de la industria cultural reitera hasta el infinito la superficie de la realidad, al hacer sonar estentóreamente la conversación comercializada y la propaganda popular cada vez más indiferenciadas una de otra ${ }^{42}$.

Así es como funciona la «cultura de masas»: fomentando la reproducción de modelos de vida social entre los individuos a través de la publicidad, donde se muestran hábitos, costumbres, formas de vida y de consumo que inoculan un modelo de vida social que derriba la verdadera e íntima individualidad.

\section{4. ¿UNA POSIBLE SALIDA A LA TRAGEDIA DE LA CULTURA MODERNA?}

Para terminar, y no tanto a modo de conclusión, dado que entendemos que la justificación de la tesis planteada ya ha sido realizada en el punto anterior, vamos a ofrecer una breve exposición de las dos posibles salidas a la situación de tragedia cultural que planteó Simmel. Si bien antes establecimos una línea de continuidad de Simmel a la Escuela de Frankfurt, ahora esta línea será regresiva, pero no hacia Hegel, sino de Simmel a Schopenhauer y Nietzsche.

Simmel plantea dos posibles vías de resolución de la tragedia cultural, pero nosotros las recogemos como una sola bajo la teoría del «doble movimiento de liberación». El objeto de esta propuesta es la restauración del carácter positivo e integral, de reconciliación, entre el sujeto y el objeto, que originariamente (al menos para Simmel) tiene la cultura. Es decir, la restauración del ideal romántico de la cultura como mediación de lo subjetivo en lo objetivo para el perfeccionamiento de ambas instancias. Las dos vías de solución que propone Simmel son la internalización de las energías vitales y la externalización de las energías vitales. El caso es que, si bien el conflicto entre la vida y la forma o la vida y la cultura es inevitable (sucedió, sucede y sucederá), es susceptible de adquirir diferentes formas de manifestación menos trágicas, o de consecuencias menos dramáticas para las sociedades humanas.

La internalización de las energías vitales (que David Frisby denomina «retiro a la ciudadela interna») parece ser la que más posibilidades de realización tiene a día de hoy, dado que se ajusta a las características sociales propias de la modernidad (aislamiento, indiferencia, reversa). Quizás por esa relación de isomorfía estructural con el "espíritu del tiempo», Adorno, que ve en ella una forma de

${ }^{42}$ Max Horkheimer, Crítica de la razón instrumental, Buenos Aires, Sur, 1973, p. 151. 
dominación, la somete a crítica. Según Adorno, el retiro a la ciudadela interna no es solución para nada, porque por mucho que el individuo que sufre la alienación productiva y cultural se parapete sobre sí mismo, es decir, se retire de la realidad, esa realidad alienante seguirá manteniendo vigencia y seguirá ejerciendo la misma opresión sobre su espíritu, que ahora solamente se manifiesta como cuerpo insensible o espíritu impasible.

Poniéndonos del lado de Adorno, consideramos que esta vía, que por cierto recuerda mucho a la propuesta de Schopenhauer en relación con el hastío y el sufrimiento que produce sobre el individuo un mundo absolutamente volitivo, no resuelve la contradicción entre la vida y la forma, sino que la suspende. Más que una solución, consiste en una evasión del problema. Se trataría simplemente de dejar al individuo arrojado al mundo en cuanto función social, y salvarlo en cuanto personalidad única. Pero resulta que el mundo moderno, según lo expone Simmel, exige más del individuo en cuanto función social que en cuanto personalidad interna, solo reservada para grupos cercanos de amistad o para la familia. La libertad que promete es una falsa libertad, apariencia de libertad. El propio Simmel parece ser consciente de ello cuando dice lo siguiente:

La salvación estética del ser, es decir, del dolor, que realiza el arte, no puede valer según su propia naturaleza más allá de los momentos de elevación estética; mientras que nos encontramos poseídos de ella, el ser y el dolor siguen existiendo en el fondo de nuestra esencia, y el intelecto, que se ha liberado de ellos por el momento, pero que no puede vivir así duraderamente, vuelve a caer en la servidumbre en que vive respecto de la voluntad ${ }^{43}$.

Por otro lado tenemos la vía de la exteriorización de las energías vitales, cuyo mejor ejemplo encuentra Simmel en el expresionismo moderno, y que podemos relacionar con la solución artística del genio que crea desde sí mismo su nueva moral superior de Nietzsche (Übermensch). Se trata de crear formas culturales no según una ley externa al individuo e impuesta por la industria cultural o por los propios productos culturales, sino de crear según una ley individual. Como lo explica Gil Villegas en Los profetas y el mesías, se trata de «exteriorizar la vida mediante una negación radical de las formas artísticas existentes ${ }^{44}$, siguiendo las nuevas corrientes futuristas y expresionistas que comienzan despuntar en los primeros años del siglo xx.

Nosotros rechazamos la vía de la interiorización siempre y cuando no sea compensada por la vía de la exteriorización. Por eso defendemos el «doble movimiento de liberación", que consiste en la retirada a la ciudadela interna para la construcción de valores, ideas y creencias que no estén alienadas por la industria de turno, pero que luego se exterioricen artísticamente para así configurar realidad no alienada, realidad que nace de uno mismo, y que por eso es capaz de expresar la indivi-

${ }^{43}$ Georg Simmel, Schopenhauer y Nietzsche, Buenos Aires, Prometeo, 2005, pp. 113-114.

${ }^{44}$ Francisco Gil Villegas, Los problemas y el mesias, México, Fondo de Cultura Económica, 1996, p. 161. 
dualidad de su sujeto creador. Un sujeto que, volviendo a Nietzsche, por cuanto es capaz de ecualizar su subjetividad con la objetividad que crea, es un genio. Según los términos orteguianos, requerimos el ensimismamiento como condición de posibilidad para una alteración consciente, autónoma y responsable. Asimismo, como criterio de genialidad y creatividad artística.

Por último, el doble movimiento de liberación recupera el carácter revolucionario del arte, que, como dice Marcuse en esta cita, ha sido desbaratado por el proceso de industrialización de la cultura moderna: «Es bueno que casi todo el mundo pueda tener ahora las bellas artes al alcance de la mano apretando tan sólo un botón en su aparato o entrando en un supermercado. En esta difusión, sin embargo, las bellas artes se convierten en engranajes de una máquina cultural que reforma su contenido $»^{45}$.

Recibido: julio de 2020; ACEPTAdo: diciembre de 2020

${ }^{45}$ Herbert Marcuse, El hombre unidimensional, Barcelona, Seix Barral, 1969, p. 95. 